\title{
,

\section{International Journal of}

Research Article

\section{Pediatrics, Neonatology and Primary Care}

\section{The role of Delayed Cord Clamping in improving the Outcome in Preterm Babies}

\author{
Dina Kamal Sayed Ali ${ }^{1 \star}$ and Hamdy Mohamed Talkhan ${ }^{2}$ \\ ${ }^{1}$ Assistant Professor of Pediatrics, Faculty of Medicine, Cairo University, Egypt \\ ${ }^{2}$ Department of Obstetrics and Gynecology, El Sahel Teaching Hospital, Cairo, Egypt
}

\section{Article Info}

\section{*Corresponding author:}

\section{Dina Kamal Sayed Ali}

Assistant professor of pediatrics

Cairo University

Eygpt

E-mail: dinakamal_2009@yahoo.com

Received: January 1, 2019
Accepted: February 18, 2019
Published: February 22, 2019

Citation: Sayed Ali DK, Talkhan HM. The role of Delayed Cord Clamping in improving the Outcome in Preterm Babies. Int J Pediatr Neonatal Prim Care. 2019; 1(1): 6-10. doi: 10.18689/ijpn-1000103

Copyright: (c) 2019 The Author(s). This work is licensed under a Creative Commons Attribution 4.0 International License, which permits unrestricted use, distribution, and reproduction in any medium, provided the original work is properly cited.

Published by Madridge Publishers

\begin{abstract}
Objective: Despite significant proposed benefits, delayed umbilical cord clamping (DCC) is not practiced widely in preterm infants largely because of the question of feasibility of the procedure and uncertainty regarding the magnitude of the reported benefits, especially intraventricular hemorrhage (IVH) vs the adverse consequences of delaying the neonatal resuscitation. The objective of this study was to determine whether implementation of the protocol-driven DCC process in Elsahel teaching hospital would improve the outcome in preterm infants without adverse consequences.
\end{abstract}

Study design: We implemented a quality improvement process for DCC for 13 months started in August 2014 in infants born at less than 37 weeks' gestational age. Eligible infants were left attached to the placenta for 45 seconds after birth. Neonatal process and outcome data were collected until discharge. We compared infants who received DCC with infants who were born for 13 months started at February 2014, but whose cord was clamped immediately after birth, because they were born before the protocol implementation.

Results: DCC was performed on 79 infants; and 81 infants were identified as control. Gestational age, birth weight, and other demographic variables were similar between both groups. There were no differences in Apgar scores or admission temperature, but significantly fewer infants in the DCC group were intubated in delivery room, had respiratory distress syndrome, or received red blood cell transfusions in the first week of life compared with the control group. A significant reduction was noted in the incidence of IVH in the DCC compared with the historic control group, after adjustment for gestational age, an association was found between the incidence of IVH and DCC. IVH was significantly lower in the DCC compared with control group. There were no significant differences in deaths and other major morbidities.

Conclusion: DCC, as performed in our institution, was associated with significant reduction in IVH and early red blood cell transfusions. DCC in preterm infants appears to be safe, feasible, and effective with no adverse consequences.

Keywords: Delaying Umbilical Cord Clamping; Intra Ventricular Hemorrhage; Preterm Infant.

\section{Introduction}

Before the mid-1950s, the term early clamping was defined as umbilical cord clamping within $1 \mathrm{~min}$ of birth, and late clamping was defined as cord clamping more than 5 minutes after birth $[1,2]$. Because of the several studies done on blood volume change after birth, which reported that $80-100 \mathrm{ml}$ of blood transfer from the placenta to the newborn in the 
first 3 minutes after birth, and up to $90 \%$ of that blood volume transfer was achieved within the first few breaths in healthy term infants, the time between birth and cord clamping was shortened to be 15-20 seconds after birth [3-5].

But recently several systematic reviews have suggested that clamping the umbilical cord in all births should be delayed for atleast 30-60 seconds, with the infant maintained at or below the level of the placenta because of the associated neonatal benefits, including increased blood volume, reduced need for blood transfusion, decreased incidence of intracranial hemorrhage in preterm infants, and lower frequency of iron deficiency anemia in term infants $[2,4,6,7]$.

WHO recommendations 2012, based on several randomized controlled studies, for basic newborn resuscitation are, in newly born babies who do not require positive-pressure ventilation, the cord should not be clamped earlier than 1 min after birth [6].

The aim of our study is to compare between immediate and delayed cord clamping in preterm infants less than 37 weeks, and its effect on the outcomes of such babies.

\section{Aim of the Study}

To study the effect of delayed cord clamping on premature infants.

\section{Patients and Methods}

On average, our level III Neonatal Intensive Care Unit cares for approximately 200 preterm inborn infants every year. The previous routine clinical practice was to clamp the umbilical cord immediately after the birth. The DCC process was implemented starting August 2014. All infants born at less than 37 weeks' gestation were eligible for DCC, unless they met the following exclusion criteria: severe maternal illness that prompted immediate delivery, placental causes (abruption or previa) or fetal causes (multiple gestation, major congenital anomalies, severe growth restriction, or hydrops fetalis). After birth, the infant was left unstimulated, attached at or slightly below the level of placenta for 45 seconds. The cord was then clamped and cut, and the neonatal team initiated resuscitation efforts. Apgar timing was initiated at the time of birth when the infant was delivered completely. We placed the baby at or below the level of the placenta as feasible. Because most of the preterm deliveries in our institution were cesarean sections, it was a challenge getting the baby truly below the level of the placenta. Because good evidence is emerging in more mature infants, the optimal timing and positioning in a very preterm infant still must be explored. A large percentage of deliveries did not receive DCC because of our predefined narrow eligibility criteria. The question of DCC being beneficial or harmful in these higher risk excluded infants (such as multiple gestations, growth restricted, and other vulnerable preterm groups) must be explored carefully in the future. We were aware of the different approaches reported in the literature like umbilical cord milking and mobile trolley use that may minimize delay in resuscitation.
The total number of babies included in the study was 160 preterm, where the protocol of DCC was applied on 79 preterm. With approval by the institutional review board, prospective and retrospective data were extracted from maternal and neonatal electronic medical records. The prospective study period was 13 months, from Aug 2014. The study period for the historic cohort was also 13 months, from February 2014. Collected data included maternal demographics, obstetric complications, any antenatal steroid and magnesium use, and other labor and delivery variables. Neonatal data included gestational age, birth weight, sex, post-delivery data variables such as Apgar scores, resuscitation data, and the infant's temperature upon admission to the neonatal intensive care unit. Other clinical variables included treatment with phototherapy, (intensive phototherapy defined as irradiance in the blue-green spectrum of at least $30 \mu \mathrm{W} / \mathrm{cm}^{2}$ per $\mathrm{nm}$ ).

For all tests of significance, $p$-values less than 0.05 were considered statistically significant and were used as evidence against the null hypothesis of no difference between DCC and ICC participants. All statistical methods were performed using the SAS software system (Release 8.2, SAS Institute, Inc., Cary, NC, USA).

\section{Results}

During the prospective study period, after implementation of DCC protocol 79 infants were born at less than 37 weeks' gestation. After excluding multiple gestation infants, DCC was performed on all of the 79 eligible infants per pre specified protocol (DCC). During the retrospective study period, 81 infants were born at less than 37 weeks' gestation, all of these infants received immediate umbilical cord clamping (ICC) after birth. There were no significant differences in maternal characteristics. Artificial reproductive therapy and cesarean delivery numbers were not different between the groups. Similarly, there were no differences in other maternal variables such as chorioamnionitis, gestational hypertension or diabetes mellitus, preeclampsia, or poly- or oligohydramnios. Overall antenatal steroid administration and maternal magnesium exposure were similar between the groups. There were no significant differences in baseline neonatal characteristics between the two groups. Mean gestational age was 34 weeks in the ICC group compared with 34.1 weeks in the DCC group; mean birth weight was $2250 \mathrm{~g}$ in the ICC cohort compared with $2325 \mathrm{~g}$ in the DCC cohort. Male infants represented $35 \%$ in DCC group, compared to $38 \%$ in the ICC group. There were no significant differences in 1- and 5-minute Apgar scores, admission temperature or $\mathrm{Ph}, \mathrm{PCO}$, $\mathrm{PO} 2$ done at birth from the umbilical cord. However, significantly fewer infants in the DCC cohort were intubated in the delivery room compared with the ICC cohort. Significant differences in glucose levels with those in the DCC group having higher initial glucose levels than those in the ICC group. Red blood cell transfusion need in the first week of life was significantly lower in the DCC cohort compared with the ICC, although the use of pressor support or corticosteroids was not different. Phototherapy in first week of life was 
significantly higher in the DCC, but none of the infants in either groups received intensive phototherapy or exchange transfusion. Incidence of RDS and surfactant administration was significantly lower in the DCC cohort. A significant reduction was noted in the incidence of IVH in the DCC group compared with the ICC group. After adjustment for gestational age, an association was found between the incidence of IVH and DCC, with IVH significantly lower in the DCC group compared with the ICC group. Also we found that, despite delaying resuscitation briefly, Apgar scores, other resuscitation variables, and mean admission temperature were not different between the DCC and ICC control groups. Additionally, a significantly lower number of infants in the DCC group were intubated in the delivery room. More infants were breathing spontaneously after DCC, which contributes to the success of non-mechanical ventilation. This supports the general hypothesis that DCC at birth decreases the need for resuscitation by promoting a more physiologic transition to extrauterine life. Number of preterm with suspected NEC (necrotizing enterocolitis) or feeding intolerance were, significantly lower in the DCC compared to ICC group.

Table 1. Maternal demographic and clinical data.

\begin{tabular}{|l|c|c|c|}
\hline Maternal Data & $\begin{array}{c}\mathrm{DCC} \\
\mathrm{n}=79\end{array}$ & $\begin{array}{c}\mathrm{ICC} \\
\mathrm{n}=81\end{array}$ & P value \\
\hline Maternal age, mean (SD) & $26.3(6.7)$ & $26.2(5.4)$ & .8 \\
\hline Artificial reproductive therapy, $\mathrm{n}(\%)$ & $2(2.5)$ & $1(1.2)$ & .8 \\
\hline Number of CS, $\mathrm{n}(\%)$ & $48(60.7)$ & $53(65 \%)$ & .9 \\
\hline Chorioamnionitis, $\mathrm{n}(\%)$ & $1(1.2)$ & $1(1.2)$ & .8 \\
\hline Gestational DM, $\mathrm{n}(\%)$ & $3(3.8)$ & $5(6 \%)$ & .66 \\
\hline Pre-eclampsia, $\mathrm{n}(\%)$ & $1(1.2)$ & $3(3.7)$ & .7 \\
\hline Poly or oligohydramnios, $\mathrm{n}(\%)$ & $5(6.3)$ & $7(8.6)$ & .9 \\
\hline Ante-natal steroid, $\mathrm{n}(\%)$ & $68(86)$ & $74(91)$ & .5 \\
\hline Mg sulfate & $1(1.2)$ & $2(2.4)$ & .8 \\
\hline
\end{tabular}

Table 2. Infants' Demographic and Clinical Characteristics after delivery.

\begin{tabular}{|l|c|c|c|}
\hline Infants Data & DCC 79 & ICC 81 & P value \\
\hline Gestational age wk, mean (SD) & $34.2(2.2)$ & $34.6(2)$ & 0.3 \\
\hline Birth Weight,g, mean (SD) & $1325(365)$ & $1250(429)$ & 0.4 \\
\hline Sex & & & \\
\hline Male, $\mathrm{n}(\%)$ & $35(44 \%)$ & $38(47 \%)$ & 0.5 \\
\hline Female, $\mathrm{n}(\%)$ & $44(56 \%)$ & $43(53 \%)$ & 0.7 \\
\hline Apgar Score at 1min, median (range) & $8(3-9)$ & $8(3-9)$ & 0.7 \\
\hline Apgar Score at 5min, median(range) & $9(4-9)$ & $9(5-9)$ & 0.7 \\
\hline Admission Temperature, mean (SD) & $36.8(2.3)$ & $36.7(2.2)$ & 0.5 \\
\hline Initial blood glucose, mean (SD) & $73(28.1)$ & $51 \mathrm{~s}(18)$ & 0.03 \\
\hline Initial mean blood pressure, mean (SD) & $29(4.8)$ & $33(6)$ & 0.1 \\
\hline Intubation in the delivery room, $\mathrm{n}(\%)$ & $3(3.8 \%)$ & $9(8.6 \%)$ & 0.03 \\
\hline $\mathrm{PH}$, mean (SD) & $7.3(0.06)$ & $7.3(0.07)$ & 0.6 \\
\hline $\mathrm{PCO}$ mmHg, mean (SD) & $47(13.2)$ & $46.3(11.6)$ & 0.7 \\
\hline PO2 mm Hg, mean (SD) & $55(28)$ & $60.3(20.4)$ & 0.8 \\
\hline
\end{tabular}

Table 3. Infant morbidity during NICU stay.

\begin{tabular}{|l|c|c|c|}
\hline Infants Morbidity & DCC 79 & ICC 81 & P value \\
\hline Blood transfusion after 1 wk of birth, $\mathrm{n}(\%)$ & $23(33)$ & $35(49)$ & 0.02 \\
\hline Pressor Support or Corticosteroid, $\mathrm{n}(\%)$ & $41(60)$ & $45(61)$ & 0.9 \\
\hline Phototherapy, $\mathrm{n}(\%)$ & $53(70)$ & $39(49)$ & 0.03 \\
\hline RDS-use of surfactant n (\%) & $41(60)$ & $63(80)$ & 0.02 \\
\hline Assisted Ventilation, n (\%) & $49(62)$ & $65(82)$ & 0.03 \\
\hline Days on oxygen, mean \pm SD & $45(14.3)$ & $49(16.7)$ & 0.3 \\
\hline IVH, $\mathrm{n}(\%)$ & $9(20)$ & $24(30)$ & 0.01 \\
\hline Suspected NEC, n (\%) & $9(7.9)$ & $14(20)$ & 0.01 \\
\hline
\end{tabular}

\section{Discussion}

There are several factors that contributed to the high rate of compliance with DCC in our institution. First, the process was developed with substantial inter professional coordination between the departments of obstetrics and neonatology that resulted in standardized protocol and narrow eligibility criteria to overcome perceived barriers. In addition, the extensive implementation plan included staff education, simulation exercises, and interim monitoring. Finally, this intervention was incorporated into our Golden Hour protocol that has objectives and processes for preterm infant care in the delivery room that we have used successfully in our practice. Our study agreed with other studies about delayed cord clamping in preterm infants. Many obstetricians and neonatologists share the same concern regarding DCC in preterm infants, which are adverse outcomes that result from delaying the resuscitation in these infants [8-12]. We found that, despite delaying resuscitation briefly, Apgar scores, other resuscitation parameters, and mean admission temperature were not different between the DCC and control group. Additionally, a significantly lower number of infants in the DCC were intubated in the delivery room. More infants were breathing spontaneously after DCC, which contributes to the success of non-mechanical ventilation. This supports the general hypotheses that DCC at birth decrease the need for resuscitation by promoting a more physiologic transition to extra uterine life $[13,14]$. Our observed reduction in the incidence of RDS and surfactant administration adds evidence to the recommendation of DCC for decreased incidence of $\operatorname{RDS}[15,16]$.

In our study, preterm babies who needed blood transfusion were lower in the DCC group compared to ICC group, this was in agreement with physiologic studies in preterm infants, which have shown that a transfer from the placenta of approximately $80 \mathrm{~mL}$ of blood occurs by 1 minute after birth, reaching approximately $100 \mathrm{~mL}$ at 3 minutes after birth. This additional blood can supply extra iron, amounting to $40-50 \mathrm{mg} / \mathrm{kg}$ of body weight. This extra iron, combined with body iron (approximately $75 \mathrm{mg} / \mathrm{kg}$ of body weight) present at birth in a preterm newborn, may help prevent iron deficiency during the first year of life $[17,18]$.

According to Mark Sloan, M.D., whether a baby "is premature or full term, approximately one-third of its total blood volume resides in the placenta. This is equal to the volume of blood that will be needed to fully perfuse the fetal lungs, liver, and kidneys at birth. In addition to the benefits that come with adequate iron stores. . babies whose cords are clamped at 2 to 3 minutes-and thus, who have an increased total blood volume compared with their immediately-clamped peers-have a smoother cardiopulmonary transition at birth $[19,20]$. Another potential benefit of delayed cord clamping is to ensure that the baby can receive the complete retinue of clotting factors." In other words, the increased volume of blood will naturally increase blood platelet levels, which are needed for normal blood clotting [21,22]. 
In our study, the number of babies who needed phototherapy in the DCC group were significantly higher than ICC group. One analysis found a very slight (2\%) increase in jaundice among babies who received delayed cord clamping. However, according to the Thinking Midwife, "The only studies available involve the administration of an artificial oxytocic (syntocinon or syntometrine) in the 'delayed clamping' group IV syntocinon is associated with jaundice. Therefore, it could be the oxytocic making a difference herenot the clamping. Other studies, found "that the difference between early and late cord clamping for clinical jaundice did not reach statistical significance. Another concern sometimes mentioned is polycythemia, or blood that is too thick to properly oxygenate tissues. Researchers also looked at this issue and did not find anything statistically significant $[23,24]$. In agreement with other studies, our study showed significantly lower number of pt with IVH and suspected NEC in DCC group. There is growing evidence that enhanced placental transfusion by delaying umbilical cord clamping (DCC) in preterm infants may improve hemodynamic stability after birth and decrease the incidence of major neonatal morbidities, such as intraventricular hemorrhage (IVH) and necrotizing enterocolitis (NEC) $[24,25]$. Delayed clamping also results in an infusion of "stem cells, which play an essential role in the development of the immune, respiratory, cardiovascular, and central nervous systems, among many other functions. The concentration of stem cells in fetal blood is higher than at any other time of life. ICC [immediate cord clamping] leaves nearly one-third of these critical cells in the placenta. Stem cells may also "help to repair any brain damage the baby might have suffered during a difficult birth," [26,27].

This study has several limitations. This study reports observational data of a process from a single center. Thus, conclusions from this study are limited to associations. Confounding of the results by other changes in practice is also a risk. However, our practice did not change much during the study period other than more widespread use of non mechanical ventilation in very preterm infants. This might have had some impact on our lower intubation and surfactant administration rates. The radiologists reading the cranial ultrasound scans were not blinded formally, but they were not aware of the implementation of DCC. There is also the question of generalizability because the data reported are from a single center. Despite these limitations, we believe it is important to share our observations, which more likely reflect the real world clinical practice and address some of the concerns that are impeding the widespread practice of DCC in preterm infants.

\section{Conclusion}

DCC, as performed in our institution, was associated with significant reduction in incidence of IVH. DCC in preterm infants appears to be safe, feasible, and effective with no adverse consequences. Our study demonstrates that implementation of the DCC process with standardized protocol in preterm infants is feasible and effective with improved outcomes. In conclusion, we have implemented DCC process successfully in a large delivery hospital. DCC, as performed in our hospital, was associated with a significant reduction in IVH and early red blood cell transfusion. Further clinical studies are needed to optimize the timing and technique of DCC and to report the impact of this potentially valuable procedure on long term neuro developmental outcomes of the preterm infants.

\section{References}

1. Ibrahim HM, Krouskop RW, Lewis DF, Dhanireddy R. Placental transfusion: umbilical cord clamping in preterm infants. J Perinatol. 2000; 20(6): 351-354.

2. Yao AC, Lind J. Placental transfusion. Am J Dis Child. 1974; 127(1): 128141. doi: 10.1001/archpedi.1974.02110200130021

3. Committee on Obstetric Practice, American College of Obstetricians and Gynecologists. Committee Opinion No. 543: Timing of umbilical cord clamping after birth. Obstet Gynecol. 2012; 120(6): 1522-1526. doi: 10.1097/01. AOG.0000423817.47165.48

4. Linderkamp O. Placental transfusion: determinants and effects. Clin Perinatol. 1982; 9(3): 559-592

5. Philip AGS, Saigal S. When should we clamp the umbilical cord? Neo Reviews. 2004; 5(4).

6. WHO. Guidelines on basic newborn resuscitation. Geneva, World Health Organization; 2012.

7. Al-Wassia $\mathrm{H}$, Shah PS. Efficacy and safety of umbilical cord milking at birth: a systematic review and meta-analysis. JAMA Pediatr. 2015; 169(1): 18-25. doi: 10.1001/jamapediatrics.2014.1906

8. Jelin AC, Kupperman M, Erickson K, Clyman R, Schulkin J. Obstetrician's attitudes and beliefs regarding umbilical cord clamping. J Matern Fetal Neonatal Med. 2014; 27(14): 1457-1461. doi: 10.3109/14767058.2013.864275

9. Farrar D, Tuffnell D, Airey R, Duley L. Care during the third stage of labour: a postal survey of UK midwives and obstetricians. BMC Pregnancy Childbirth. 2010; 10-23. doi: 10.1186/1471-2393-10-23

10. Ononeze $A B$, Hutchon DJ. Attitude of obstetricians towards delayed cord clamping: a questionnaire-based study. J Obstet Gynaecol. 2009; 29(3): 223-224. doi: 10.1080/01443610802712918

11. Reynolds GJ. Beyond sweetness and warmth: transition of the preterm infant. Arch Dis Child Fetal Neonatal Ed. 2008; 93(1): F2-F3. doi: 10.1136/ adc.2007.120790

12. Bell EF. Increasing the placental transfusion for preterm infants. Obstet Gynecol. 2011; 117(2): 203-204. doi: 10.1097/AOG.0b013e3182056828

13. Redmond $D$, Isana $S$, Ingall $D$. Relation of onset of respiration to placental transfusion. Lancet. 1965; 285(7380): 283-285. doi: 10.1016/S01406736(65)91025-1

14. Kjeldsen J. Pedersen J. Relation of residual placental blood volume to onset of respiration and respiratory distress syndrome in infants of diabetic and non-diabetic mothers. Lancet. 1967; 289(7483): 180-184. doi: 10.1016/S0140-6736(67)91823-5

15. Bound JP, Harvey PW, Bagshaw HB. Prevention of pulmonary syndrome of the newborn. Lancet. 1962; 280(7249): 1200-1203.

16. Usher RH, Saigal S, O'Neill A, Surainder Y, Chua LB. Estimation of red blood cell volume in premature infants with and without respiratory distress syndrome. Biol Neonate. 1975; 26(3-4): 241-248. doi: $10.1159 / 000240735$

17. American Academy of Pediatrics Subcommittee on Hyperbilirubinemia. Management of hyperbilirubinemia in the newborn infant 35 or more weeks of gestation. Pediatrics. 2004; 114(1): 297-316.

18. Bell MJ, Ternberg JL, Feigin RD. et al. Neonatal necrotizing enterocolitis: therapeutic decisions based upon clinical staging. Ann Surg. 1978; 187(1): $1-7$. 
19. Sommers R, Stonestreet BS, Oh W, et al. Hemodynamic effects of delayed cord clamping in premature infants. Pediatrics. 2012; 129(3): e667-e672. doi: 10.1542/peds.2011-2550

20. Rabe H, Diaz-Rossello JL, Duley L, Dowswell T. Effect of timing of umbilical cord clamping and other strategies to influence placental transfusion at preterm birth on maternal and infant outcomes. Cochrane Database Syst Rev. 2012; 15(8): CD003248. doi: 10.1002/14651858

21. Ehrenkranz RA, Walsh MC, Vohr BR. et al. Validation of the National Institutes of Health consensus definition of bronchopulmonary dysplasia. Pediatrics. 2005; 116(6): 1353-1360. doi: 10.1542/peds.2005-0249

22. Raju TN, Singhal N. Optimal timing for clamping the umbilical cord after birth. Clin Perinatol. 2012; 39(1): 889-900. doi: 10.1016/j.clp.2012.09.006

23. An International Committee for the Classification of Retinopathy of Prematurity. The international classification of retinopathy of prematurity revisited. Arch Ophthalmol. 2005; 123(7): 991-999. doi: 10.1001/ archopht.123.7.991
24. Papile LA, Burstein J, Burstein R, Koffler $H$. Incidence and evolution of subependymal and intraventricular hemorrhage: a study of infants with birth weights less than $1500 \mathrm{gm}$. J Pediatr. 1978; 92(4): 529-534.

25. Bolisetty S, Dhawan A, Abdel-Latif M, Bajuk B, Stack J, Lui K. Intraventricular hemorrhage and neurodevelopmental outcomes in extreme preterm infants. Pediatrics. 2014; 133(1): 55-62. doi: 10.1542/peds.2013-0372

26. Mercer JS, Vohr BR, McGrath MM, Padbury JF, Wallach M, Oh W. Delayed cord clamping in very preterm infants reduces the incidence of intraventricular hemorrhage and late-onset sepsis: a randomized, controlled trial. Pediatrics. 2006; 117(4): 1235-1242. doi: 10.1542/ peds.2005-1706

27. Backes $\mathrm{CH}$, Rivera $\mathrm{BK}$, Haque $U$, et al. Placental transfusion strategies in very preterm neonates: a systemic review and meta-analysis. Obstet Gynecol. 2014; 124(1): 47-56. doi: 10.1097/AOG.0000000000000324 